\title{
STURM-LIOUVILLE EQUATIONS WITH BESICOVITCH ALMOST-PERIODICITY
}

\author{
A. DZURNAK AND A. B. MINGARELLI
}

(Communicated by Kenneth R. Meyer)

\begin{abstract}
In this article we extend a former result [Proc. Amer. Math. Soc. 97, (1986), 269-272] dealing with the oscillation of (Bohr) almost-periodic Sturm-Liouville operators to the generalization of such as considered by Besicovitch. This includes all the classical extensions of almost periodic functions as considered by Stepanoff and Weyl.
\end{abstract}

\section{INTRODUCTION}

Let $V: \mathbb{R} \rightarrow \mathbb{R}$, be locally Lebesgue integrable and consider the scalar secondorder differential equation

$$
(*)-y^{\prime \prime}(x)+\lambda V(x) y(x)=0, \quad-\infty<x<+\infty,
$$

where $\lambda$ is a real nonzero coupling constant and where $V$ is a real generalized almost-periodic function in the sense of Bohr, Stepanoff, Weyl, or Besicovitch. Each such stated class of functions is included in the next one $[1$, p. 76] or $[2$, p. 95]. Bohr a.p. functions are, by definition, continuous whereas the others are not necessarily so.

The class of Besicovitch almost-periodic functions, for example, is defined as the closure of the set of all finite trigonometric polynomials in the Besicovitch seminorm, \|\|$_{B}$, (see [1] or [2] for details):

$$
\|V\|_{B}=\limsup _{T \rightarrow \infty} \frac{1}{2 T} \int_{-T}^{T}|V(s)| d s .
$$

These functions may be characterized by "translation properties", as in the Bohr case [1, p. $77 \mathrm{ff}$.]. The mean value, $M\{V\}$, of such a generalized almostperiodic function $V$ always exists, is finite, and is uniform with respect to $a$, for $-\infty<a<+\infty[1$, p. 93, Lemma 4 and Corollary 1], where

$$
M\{V\}=\lim _{T \rightarrow \infty} \frac{1}{T} \int_{0}^{T} V(s+a) d s .
$$

Received by the editors February 15, 1988 and, in revised form, May 8, 1988.

1980 Mathematics Subject Classification (1985 Revision). Primary 34C10.

Key words and phrases. Bohr-, Stepanoff-, Weyl- and Besicovitch almost-periodic functions.

This research was partially funded by a NSERC-Canada grant 
It is immediate from the translation properties of such functions that $M\{|V|\}$ always exists, since $|V|$ enjoys the same almost-periodic properties as does $V$, and is finite [1, p. 94, Lemma 5].

In a previous paper [4], it was shown that under the assumption that $V$ is real Bohr almost-periodic, $V$ not identically zero, the equation (1.1) is oscillatory at bot $h+\infty$ and $-\infty$ for every real $\lambda$ not zero if and only if $M\{V\}=0$. This was extended slightly in [7] to include the case of generalized almost-periodic functions in the sense of Stepanoff, under the tacit assumption that either $V$ or its indefinite integral is uniformly bounded on $R$.

In each of the cited works use is made of the Bohr uniqueness theorem whereby a non-negative a.p. function with mean value equal to zero must vanish identically, if it is Bohr a.p., or vanish a.e. with respect to Lebesgue measure, if it is Stepanoff a.p. It is a classical result that this uniqueness theorem fails in the Weyl or Besicovitch case.

It turns out that a natural (a priori) condition, namely, that $M\{|V|\}>0$, is needed in addition to the original one, that is, $M\{v\}=0$, in order to extend the result of [4] to the Stepanoff, Weyl and Besicovitch cases, and that the former condition is necessary. This is seen by the counterexample to the sufficiency, if $M\{|V|\}=0$ and $M\{V\}=0$, at the end of $\S 2$.

Equation (1.1) is said to be oscillatory at $+\infty$ and $-\infty$ if every solution has an infinity of zeros accumulating only at $+\infty$ and $-\infty$. It is said to be nonoscillatory on $[a, \infty)$ if every nontrivial solution has only finitely many zeros there.

Furthermore, it is known that if $V$ is Bohr a.p. then the equation

$$
y^{\prime \prime}+V(x) y=0, \quad x \in \mathbf{R}
$$

is either oscillatory at $+\infty$ and $-\infty$ or else is disconjugate on $\mathbf{R}$, see $[6$, Lemma 1] or [3, p. 25, proof of Theorem 14]. This result has been extended to the Stepanoff a.p. case in [7, p. 48, Theorem 2.7.1]. We show here that this is, in general, false for Weyl a.p. functions, see the end of $\S 2$. We note, in passing, that the class of all Weyl a.p. functions is quite large and includes $L(\mathbf{R})$. See $[1$, p. 77] for the definition of the former class.

2. We prove the following results:

Theorem 1. Let $V$ be Besicovitch almost-periodic and assume that

$$
M\{|V|\}>0 .
$$

Then (1.1) is oscillatory at $+\infty$ and $-\infty$ for every real $\lambda$ not equal to zero if and only if $M\{V\}=0$.

We postpone the proof until $\S 3$.

Corollary 1. Let $V$ be Stepanoff a.p., $V(x)$ not a.e. zero.

Then (1.1) is oscillatory at $+\infty$ and $-\infty$, for every real $\lambda$ not zero, if and only if $M\{V\}=0$. 
Of course, the proof of this result is an immediate consequence of the Stepanoff uniqueness theorem, see e.g., [7], and Theorem 1. Since every Bohr a.p. function is Stepanoff a.p. this corollary includes the main result in [4].

Now the function

$$
V(x)=\exp \left(-x^{2}\right), \quad-\infty<x<+\infty,
$$

is Weyl a.p., [1, p. 77] and $M\{V\}=0=M\{|V|\}$. Moreover, the equation (1.1) is nonoscillatory on $(-\infty,+\infty)$ if $\lambda>0$. This shows that $(2.1)$ cannot be waived, in general.

Next, let $V$ be defined on $\mathbb{R}$ as follows: On $[0, \infty), V(x)=1 / 2(x+1)^{2}$, while on $(-\infty, 0], V(x)=1 / 8(x-1)^{2}$. Since each of these $V$ gives rise to an Euler equation on the respective half-axes, we see that the resulting equation is oscillatory on $[0, \infty)$ and nonoscillatory on $(-\infty, 0]$. Moreover, $V$ is Weyl a.p. as it is in $L(\mathbb{R})$.

3. The proof of the main theorem is based upon the following interesting comparison theorem of Levin [5], and some results of independent interest.

Lemma 1. Let $\varphi_{i}:[a, b] \rightarrow \mathbb{R}$, be in $L(a, b)$ for $i=1,2$. If $x_{1}(t)$ is a solution of the equation

$$
x^{\prime \prime}+\varphi_{1}(t) x=0, \quad t \in[a, b]
$$

which does not vanish on $[a, b]$ and for each $t$ in $[a, b]$ there holds

$$
-x_{1}^{\prime}(a) / x_{1}(a)+\int_{a}^{t} \varphi_{1}(s) d s>1-x_{2}^{\prime}(a) / x_{2}(a)+\int_{a}^{t} \varphi_{2}(s) d s \mid
$$

for some solution $x_{2}$ of the equation

$$
x^{\prime \prime}+\varphi_{2}(t) x=0, \quad t \in[a, b]
$$

then $x_{2}(t)$ does not vanish on $[a, b]$ and

$$
-x_{1}^{\prime}(t) / x_{1}(t)>\left|x_{2}^{\prime}(t) / x_{2}(t)\right|, \quad t \in[a, b]
$$

Proof. This is actually given in [5, Theorem 1].

Corollary 2. Let $V_{i}:[a, b] \rightarrow R$ satisfy the conditions of the lemma, for $i=1,2$. Let $z_{i}(t)$ denote the solutions of the Riccati equation

$$
z^{\prime}(t)-z^{2}(t)+V_{i}(t)=0, \quad t \in[a, b] .
$$

Assume that $z_{1}(t)$ is finite on $[a, b]$. If for each $t$ in $[a, b]$ there holds

$$
z_{1}(a)-\int_{a}^{t} V_{1}(s) d s>\left|z_{2}(a)-\int_{a}^{t} V_{2}(s) d s\right|
$$

then $z_{2}(t)$ is also finite on $[a, b]$ and

$$
z_{1}(t)>\left|z_{2}(t)\right|, \quad t \in[a, b] .
$$

Proof. This is essentially an immediate consequence of the lemma as is seen by setting $\varphi_{i}=-V_{i}$, and $z_{i}^{\prime}(t)=-x_{i}^{\prime}(t) / x_{i}(t)$. 
Lemma 2. Let $V:[0, \infty) \rightarrow R$ be locally integrable. Assume that $V$ has a mean value, $M\{V\}$, defined above, and $M\{V\}=0$.

If $z(x)$ is a finite solution of the equation

$$
z^{\prime}-z^{2}+V=0, \quad \text { for } x \geq 0,
$$

then

$$
\lim _{T \rightarrow \infty} \frac{1}{T} \int_{0}^{T} z^{2}(s) d s=0,
$$

Proof. It suffices to show that

$$
\limsup _{T \rightarrow \infty} \frac{1}{T} \int_{0}^{T} z^{2}(s) d s=0
$$

Assume the contrary. Applying (3.1) to $[0, T]$, we have,

$$
z(T) / T=z(0) / T-(1 / T) \int_{0}^{T} V(s) d s+(1 / T) \int_{0}^{T} z^{2}(s) d s
$$

holding for each $T \geq 0$. Since $M\{V\}=0$ the initial assumption implies the existence of a sequence $\left\{T_{n}\right\}, T_{n} \rightarrow \infty$, for which

$$
z\left(T_{n}\right) / T_{n}>m^{2}, \text { where } m>0 .
$$

Furthermore, we have

$$
\left|\int_{0}^{T} V(s) d s\right|<m^{2} T / 4, \quad T>T_{0},
$$

for $T_{0}$ sufficiently large. Hence, for $x \geq T_{n} \geq T_{0}$, a double application of (3.4) shows that,

$$
\int_{T_{n}}^{x} V(s) d s<\left(x+T_{n}\right) m^{2} / 4=T_{n} m^{2} / 2+\int_{T_{n}}^{x} m^{2} / 4 d s .
$$

The latter implies that

$$
z\left(T_{n}\right)-\int_{T_{n}}^{x} V(s) d s>z\left(T_{n}\right)-T_{n} m^{2} / 2-\int_{T_{n}}^{x} m^{2} / 4 d s \geq 0,
$$

provided $\left[z\left(T_{n}\right)-T_{n} m^{2} / 4\right] /\left(m^{2} / 4\right) \geq x \geq T_{n}>T_{0}$. Since $z\left(T_{n}\right)>T_{n} m^{2}$, the former inequality is verified whenever $3 T_{n} \geq x \geq T_{n}>T_{0}$. In order to use Levin's comparison theorem, we compare the equations (3.1) and

$$
z^{\prime}-z^{2}+\left(m^{2} / 4\right)=0, \quad \text { on }\left[T_{n}, 3 T_{n}\right],
$$

where $T_{n}>T_{0}$. Let $z_{2}(t)$ be a solution of (3.6) satisfying the initial condition

$$
z_{2}\left(T_{n}\right)=z\left(T_{n}\right)-T_{n} m^{2} / 2 \text {. }
$$

Then applying (3.5) and Corollary 2, we find that $z_{2}$ is finite on $\left[T_{n}, 3 T_{n}\right]$ for each sufficiently large $n$. But since $z\left(T_{n}\right)>T_{n} m^{2}$ we also have $z_{2}\left(T_{n}\right) \rightarrow \infty$, as $n \rightarrow \infty$. Moreover,

$$
z(x) \geq\left|z_{2}(x)\right|, \quad \text { on }\left[T_{n}, 3 T_{n}\right] .
$$


We now compare the solution of (3.6) satisfying (3.7) with a solution, $z_{3}(x)$, of the differential inequality

$$
z^{\prime}-z^{2}+\left(m^{2} / 4\right)<0, \quad \text { on }\left[T_{n}, 3 T_{n}\right],
$$

for which $z_{3}\left(T_{n}\right)=z_{2}\left(T_{n}\right)$. A standard comparison theorem now shows that

$$
z_{2}(x) \geq z_{3}(x), \quad \text { on }\left[T_{n}, 3 T_{n}\right],
$$

where $z_{3}(x)$ is given by

$$
z_{3}(x)=m / 2+1 /\left(T_{n}-x+1 /\left[z_{2}\left(T_{n}\right)-m / 2\right]\right) .
$$

Now, if $n$ is sufficiently large, the point

$$
T_{n}+1 /\left[z_{2}\left(T_{n}\right)-m / 2\right] \in\left[T_{n}, 3 T_{n}\right]
$$

since $z_{2}\left(T_{n}\right) \rightarrow \infty$ with $n$. Thus, for such $n$, it follows that there exists an $x \in\left[T_{n}, 3 T_{n}\right]$, at which $z_{3}(x)$ becomes unbounded, i.e., $z_{2}(x)$ cannot be finite on $\left[T_{n}, 3 T_{n}\right]$ for such $n$, on account of (3.8). This, in turn, implies that $z(x)$ must become unbounded on such an interval for each sufficiently large $n$, which is a contradiction. This completes the proof of the lemma.

Proof of Theorem 1. The necessity is proved in [4] where the theorem is stated for $V$ a Bohr almost-periodic function. The proof is valid for the class of Besicovitch almost-periodic functions with no change (see [4, Remark]), since the necessity requires merely the "uniformity" of the mean value as stated at the outset.

Sufficiency. It suffices to show that (1.1), with $\lambda=1$, is oscillatory on $R$. If $V$ is Bohr almost periodic the proof can be found in [6, Theorem 4] or [3, Theorem 14] or [7, p. 32, Theorem 2.5.12]. Let us assume, on the contrary, that $(1.1)$, with $\lambda=1$, has a solution $y$ that is nonoscillatory at $+\infty$ or $-\infty$. Without loss of generality we can suppose that $y$ is nonoscillatory at $+\infty$ and that $y$ is positive on $[0, \infty)$ (as the choice of the left end-point is immaterial in what follows).

Setting $z=-y^{\prime} / y$ we see that $z$ satisfies the Riccati equation (3.1) for $x \geq 0$, and that $z$ is finite for any $x \geq 0$. Let $\delta>0$ be an arbitrary constant. Then

$$
(1 / \delta) \int_{x}^{x+\delta} V=(1 / \delta) \int_{x}^{x+\delta} z^{2}-z(x+\delta) / \delta+z(x) / \delta
$$

We now apply the natural Besicovitch seminorm, \|\|$_{B^{\prime}}$, essentially a restriction of \|\|$_{B}$ to a semiaxis, defined by

to find

$$
\|f\|_{B^{\prime}}=\limsup _{T \rightarrow \infty}(1 / T) \int_{0}^{T}|f(s)| d s
$$

$$
\begin{aligned}
0 \leq\left\|(1 / \delta) \int_{x}^{x+\delta} V\right\|_{B^{\prime}} \leq & \left\|(1 / \delta) \int_{x}^{x+\delta} z^{2}\right\|_{B^{\prime}} \\
& +\|z(x+\delta) / \delta\|_{B^{\prime}}+\|z(x) / \delta\|_{B^{\prime}}
\end{aligned}
$$


Since $M\{V\}=0$, Lemma 2 shows that $M\left\{z^{2}\right\}=0$, and consequently, $\|z\|_{B^{\prime}}=0$. It now follows that also $\|z(x+\delta)\|_{B^{\prime}}=0$, for each $\delta>0$.

Now, a change of variable in the inner integral followed by an application of Fubini's theorem shows that, for each $\delta>0$,

$$
\begin{aligned}
(1 / T \delta) \int_{0}^{T} \int_{s}^{s+\delta} z^{2}(r) d r d s & =(1 / T \delta) \int_{0}^{\delta} \int_{0}^{T} z^{2}(r+s) d s d r \\
& \leq(1 / T \delta) \int_{0}^{\delta} \int_{0}^{T+\delta} z^{2}(s) d s d r \\
& \leq(1 / T) \int_{0}^{T+\delta} z^{2}(s) d s
\end{aligned}
$$

Passing to the limit as $T \rightarrow \infty$, we see that, for each $\delta>0$,

$$
\left\|(1 / \delta) \int_{x}^{x+\delta} z^{2}(s) d s\right\|_{B^{\prime}}=0
$$

since $M\left\{z^{2}\right\}=0$. Applying the foregoing results to (3.9) we find that for each $\delta>0$,

$$
\left\|(1 / \delta) \int_{x}^{x+\delta} V(s) d s\right\|_{B^{\prime}}=0
$$

Since $V$ is Besicovitch almost periodic [1, p. 97, Lemma],

$$
\lim _{\delta \rightarrow 0}\left\|V(x)-(1 / \delta) \int_{x}^{x+\delta} V(s) d s\right\|_{B^{\prime}}=0
$$

A combination of the last two results shows that

$$
\|V\|_{B^{\prime}}=0 \text {. }
$$

But since $M\{|V|\}=\|V\|_{B^{\prime}}$, we get the desired contradiction.

We note, in passing, that the choice of seminorm used above, namely the $B$ - or $B^{\prime}$-seminorm is a matter of indifference since either one generates the same space of Besicovitch a.p. functions (see [1] or [2]). thus the $B^{\prime}$-seminorm could well have been used in the definition at the outset rather than our $B$ seminorm.

\section{ACKNOWLEDGMENT}

The authors are grateful to Professor James S. Muldowney, of the University of Alberta, for bringing to our attention, in 1984, the work by Yu. Levin [5].

\section{REFERENCES}

1. A. Besicovitch, Almost-periodic functions, Dover, New York, 1954, xiii, 180 p.

2. H. Bohr, Almost-periodic functions, Chelsea, New York, 1951, 114p.

3. W. Coppel, Disconjugacy, Lecture Notes in Mathematics-220, Springer-Verlag, New York, $1971,148 \mathrm{p}$. 
4. S. G. Halvorsen and A. B. Mingarelli, On the oscillation of almost-periodic Sturm-Liouville operators with an arbitrary coupling constant, Proc. Amer. Math. Soc. 97 (1986), 269-272.

5. A. Yu. Levin, A comparison principle for second order differential equations, Soviet Math. Dokl. 1, (1960), 1313-1316.

6. L. Markus and R. A. Moore, Oscillation and disconjugacy for linear differential equations with almost-periodic coefficients, Acta Math. 96, (1956), 79-123.

7. A. B. Mingarelli and S. G. Halvorsen, Non-oscillation domains of differential equtins with two parameters, Lecture Notes in Mathematics, Springer-Verlag, New York, Vol. 1338, (1988).

Department of Mathematics, The University of Ottawa, Ottawa, Ontario, Canada, K1N 6N5 